\title{
Asiaticoside improves spatial learning and memory abilities of Alzheimer's disease rat model by promoting the expression of PPAR $\gamma$ and inhibiting inflammatory factors.
}

\author{
Xian Jiang1, Siwei Chen ${ }^{2}$, Fang Wang ${ }^{3}$, Daqiang Song ${ }^{4}$, Hong Zhang ${ }^{4}$, Zhuo Zhang ${ }^{\text {* }}$ \\ ${ }^{1}$ Department of Anesthesiology, the Affiliated Hospital of Southwest Medical University, Luzhou, PR China \\ ${ }^{2}$ Department of Traditional Chinese Medicine Preparation, School of Pharmacy, Southwest Medical University, Luzhou, \\ PR China \\ ${ }^{3}$ Department of Pharmaceutical Analysis, School of Pharmacy, Southwest Medical University, Luzhou, PR China \\ ${ }^{4}$ Department of Pharmacology, School of Pharmacy, Southwest Medical University, Luzhou, PR China
}

\begin{abstract}
Aims: The present study is to investigate the effect of Asiaticoside (AS) on the expression of hippocampal Peroxisome Proliferator Activated Receptor Gamma (PPAR $\gamma$ ) and inflammatory factors such as Interleukin (IL)-6 and Tumor Necrosis Factor (TNF)- $\alpha$ in rats after intracerebral injection of A $\beta 1$ 1-42. Methods: A total of 40 specific pathogen-free Sprague-Dawley rats were evenly divided into shamoperation (control) group, model group, model+AS $(15 \mathrm{mg} / \mathrm{kg})$ group and model+AS $(45 \mathrm{mg} / \mathrm{kg})$ group. Condensed $\beta$-amyloid protein $(\mathrm{A} \beta)-1-42$ was slowly injected into the CA1 region in hippocampus of rats to simulate Alzheimer's disease. Morris water maze test was used to examine the ability of spatial learning and memory of rats. Immunohistochemistry was performed to detect the deposition of A $\beta 1-42$ in hippocampal tissues. Enzyme-linked immunosorbent assay was carried out to determine the contents of IL-6 and TNF- $\alpha$ in hippocampal tissues. Western blotting was employed to measure the protein expression of PPAR $\gamma$.

Results: Treatment with AS enhanced the spatial learning and memory, reduced the expression of A $31-42$ in hippocampal region, decreased the contents of IL-6 and TNF- $\alpha$ in hippocampal tissues, and elevated the protein expression of PPAR $\gamma$ in hippocampal tissues of AD model rats.

Conclusions: The present study demonstrates that AS improves spatial learning and memory abilities of AD model rats by promoting the expression of $\operatorname{PPAR} \gamma$ and inhibiting the contents of inflammatory factors.
\end{abstract}

Keywords: Asiaticoside, Peroxisome proliferator activated receptor- $\gamma$, Inflammation, Alzheimer's disease. Accepted on January 3, 2018

\section{Introduction}

The pathogenesis of Alzheimer's Disease (AD) is complex and is still unclear. Inflammatory responses play important roles in the pathogenesis of $\mathrm{AD}$. Immunological study shows that a large number of complements exist in the focus of $\mathrm{AD}$ and the sources of the complements in the brain are astrocytes and microglia. Inflammation and oxidative stress induced by $\beta$ amyloid protein $(A \beta)$ are important factors for the occurrence of Alzheimer's Disease (AD) [1]. A $\beta$ is composed of 39-43 amino acids, and aggregated $A \beta$ can induce neuronal cell apoptosis and damage neuronal cells [2]

Peroxisome Proliferator Activated Receptor Gamma (PPAR- $\gamma$ ) is a ligand-activated nuclear transcription factor that regulates the metabolism of glucose and lipid and inhibits the expression of inflammatory genes [3]. The activation of PPAR- $\gamma$ promotes the differentiation and maturation of neural cells, participates in the programmed death of neuronal cells, and is correlated with the inflammation and oxidative stress in neural cells [4]. PPAR- $\gamma$ inhibits the expression of pro-inflammatory genes at the transcriptional level by antagonizing the activities of transcription factors NF- $\mathrm{B}, \mathrm{AP}-1$ and STAT1. Its agonists, such as pioglitazone, can effectively inhibit the production of inflammatory molecules mediated by medium and small glial cells and astrocytes in the central nervous system. Under normal physiological conditions, the expression of PPAR $\gamma$ in the brain is low, but the levels of PPAR $\gamma$ in the brain of AD patients are significantly elevated [5].

Asiaticoside (AS), extract of Centella asiatica, has antiinflammatory effect and reduces apoptosis of nerve cells [6]. However, the effect of AS on the expression of PPAR- $\gamma$ in rats 
with intracerebral injection of $A \beta 1-42$ is unclear yet. In the present study, we use Morris water maze test, immunohistochemistry, ELISA and Western blotting to investigate the effect of AS on the expression of hippocampal PPAR $\gamma$ and inflammatory factors such as Interleukin (IL)- 6 and Tumor Necrosis Factor (TNF)- $\alpha$ in rats after intracerebral injection of $A \beta 1-42$.

\section{Materials and Methods}

\section{Animals}

A total of 40 specific pathogen-free Sprague-Dawley rats (12 months old) with gender ratio of $1: 1$ and body weight between 300 and $400 \mathrm{~g}$ were obtained from Animal Experimental Center of Southwest Medical University (production certificate No. SCXK (Chuan) 2013-17; use license No. SCXK (Chuan) 2013-065). To construct AD model, the rats were anaesthetized by $3 \%$ pentobarbital sodium at a dose of $30 \mathrm{mg} / \mathrm{kg}$ and fixed on a brain stereotactic apparatus (Huaibei Zhenghua Biological Instrument Co., Ltd., Huaibei, China) in prostrate position. The head was incised in the middle and bregma was exposed. The skull was drilled at the position (AP, $3.5 \mathrm{~mm}$; ML, $2.0 \mathrm{~mm}$; H, $2.7 \mathrm{~mm}$ ) according to the atlas of brain stereotactic apparatus [7], and $5 \mu \mathrm{l}$ condensed $\mathrm{A} \beta 1-42(2 \mu \mathrm{g} / \mu \mathrm{l})$ was slowly injected into the CA1 region in hippocampus. Sham-operation group of rats were treated with equal amount of sterile saline. The injection time was $5 \mathrm{~min}$ and needle retaining time was $5 \mathrm{~min}$. After local disinfection, the skin was sutured. The 40 rats were evenly divided into sham-operation (control) group, model group, model+AS $(15 \mathrm{mg} / \mathrm{kg})$ group and model+AS (45 $\mathrm{mg} / \mathrm{kg}$ ) group. At $2 \mathrm{~h}$ before modeling, the rats in model $+\mathrm{AS}$ groups received the first gastric administration, and those in sham-operation group and model group received the same amount of saline. The rats were treated once every morning for consecutive two weeks. All animal experiments were conducted according to the ethical guidelines of Southwest Medical University.

\section{Morris water maze test}

Two weeks after treatment, the rats were subjected to Morris water maze test [3] using MT-200 rat Morris water maze video tracking analysis system (Taimeng Technology Co., Ltd., Chengdu, China). First, place navigation test was performed by putting the head of the rat into the water and recording the time (s) for the animal to look for the underwater platform. The animals were pretrained for finding the platform, and if the time exceeded $60 \mathrm{~s}$, the rats were guided to the platform. After the experiment was finished, the rats were dried and placed under the $150 \mathrm{~W}$ incandescent lamp to be dried for $5 \mathrm{~min}$. On the first day, the rats swam freely for $2 \mathrm{~min}$. From the second to the fifth day, the rats were trained in each quadrant once a day. The time for the rats to find and climb up the platform (escape latent period) was recorded on each day and averaged. Second, spatial search test was performed. On the fifth day, the platform was removed, and the number of times for the rats to cross the original position of the platform within 2 min was recorded.

\section{Immunohistochemistry}

After being anaesthetized, the rats were infused with formaldehyde, and hippocampal tissues were fixed in $4 \%$ paraformaldehyde for $24 \mathrm{~h}$ to obtain paraffin sections. After dewaxing, the sections were incubated with 3\% hydrogen peroxide for $10 \mathrm{~min}$, blocked with $5 \%$ bovine serum albumin for $10 \mathrm{~min}$ at room temperature, and incubated with rabbit antirat A $\beta 1-42$ (1:500; Bioss, Beijing, China) at $4^{\circ} \mathrm{C}$ overnight. Then, the sections were incubated with goat anti-rabbit secondary antibody at room temperature for $40 \mathrm{~min}$, followed by incubation with strept avidin-biotin complex at room temperature for $30 \mathrm{~min}$. After color development by DAB, the sections were counterstained by hematoxylin before mounting with resinene. The expression of A $\beta 1-42$ was observed under a microscope (XDC-10A-530HS; Suzhou Beitejia Photoelectric Technology Co., Ltd., Suzhou, China).

\section{Enzyme-linked immunosorbent assay (ELISA)}

Hippocampal tissues on the right side were collected from rats on ice. The tissues $(50 \mathrm{mg})$ were homogenized at $1500 \mathrm{X} \mathrm{g}$ at $4^{\circ} \mathrm{C}$ for $10 \mathrm{~min}$. ELISA was performed according to the manual of the manufacturer (Jingmei Biotechnology, Shenzhen, China). The absorbance for IL-6 and TNF- $\alpha$ was read at $450 \mathrm{~nm}$ on a reader (SpectraMax M3; Molecular Devices, Sunnyvale, CA, USA).

\section{Western blotting}

Hippocampal tissues $(10 \mathrm{mg})$ were lysed in $100 \mu \mathrm{l}$ cell lysis buffer containing $1 \mu \mathrm{l}$ phenylmethanesulfonyl fluoride and protease inhibitor for $10 \mathrm{~min}$. After lysis, the samples were centrifuged at $12,000 \mathrm{X} \mathrm{g}$ for $10 \mathrm{~min}$ at $4{ }^{\circ} \mathrm{C}$. The supernatant was used to determine protein concentration by bicinchoninic acid protein concentration determination kit (RTP7102, RealTimes Biotechnology Co., Ltd., Beijing, China). After boiling for $10 \mathrm{~min}$, the samples were centrifuged at $12,000 \mathrm{X} \mathrm{g}$ for 3 min at $4{ }^{\circ} \mathrm{C}$. Afterwards, $10 \mu \mathrm{l}$ samples were subject to sodium dodecyl sulfate-polyacrylamide gel electrophoresis at $10 \mathrm{~mA}$ for $30 \mathrm{~min}$, and then $100 \mathrm{~V}$. Afterwards, the resolved proteins were transferred to polyvinylidene difluoride membranes on ice $(100 \mathrm{~V}, 80 \mathrm{~min})$ and blocked with $50 \mathrm{~g} / \mathrm{L}$ skimmed milk at room temperature for $1 \mathrm{~h}$. Then, the membranes were incubated with polyclonal rabbit anti-rat PPAR $\gamma$ and $\beta$-actin antibodies (1:1000; Abcam, Cambridge, MA, USA) at $4{ }^{\circ} \mathrm{C}$ overnight. After extensive washing with phosphate-buffered saline with Tween 20 for 3 times of $15 \mathrm{~min}$, the membranes were incubated with goat anti-rabbit horseradish peroxidaseconjugated secondary antibody (1:1000; Abcam, Cambridge, MA, USA) for $1 \mathrm{~h}$ at room temperature before washing with phosphate-buffered saline with Tween 20 for 3 times of 15 min. Then, the membrane was developed with enhanced chemiluminescence detection kit (Sigma-Aldrich, St. Louis, MO, USA) for imaging. Image-Pro Plus v6.0 (Media Cybernetics, Rockville, MD, USA) software was used to 
Asiaticoside improves spatial learning and memory abilities of Alzheimer's disease rat model by promoting the expression of PPAR? and inhibiting inflammatory factors

acquire and analyse imaging signals. The relative content of PPAR $\gamma$ protein was expressed as PPAR $\gamma / \beta$-actin ratio.

\section{Statistical analysis}

The results were analysed using SPSS19.0 statistical software (IBM, Armonk, NY, USA). Measurement data were expressed as means \pm standard deviations. Data were analysed using single factor analysis of variance. Intergroup comparison was carried out using Student-Newman-Keuls test. $\mathrm{P}<0.05$ was considered statistically significant.

\section{Results}

\section{Treatment with AS enhances the spatial learning and memory of $A D$ model rats}

To examine the effect of AS on spatial learning and memory of rats, Morris water maze test was performed. The data showed that the mean latent period of rats in model group was significantly longer than that in sham-operation group $(\mathrm{P}<0.01)$, and the number of times to cross the platform in model group was significantly decreased than that in shamoperation group $(\mathrm{P}<0.01)$ (Figures 1 and 2$)$. After treatment with AS (15 mg/kg and $45 \mathrm{mg} / \mathrm{kg})$, the mean latent period was significantly decreased and the number of times to cross the platform was significantly increased compared with those in model group (both $\mathrm{P}<0.05$ ) (Figures 1 and 2). The results suggest that treatment with AS enhances the spatial learning and memory of AD model rats.

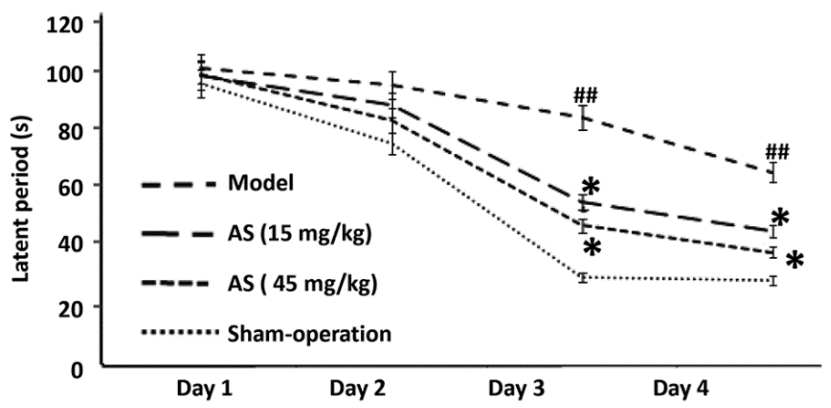

Figure 1. Effect of asiaticoside on the latent period of Alzheimer's disease model rats. Using Morris water maze, place navigation test was performed. On the first day, the rats swam freely for 2 min. From the second to the fifth day, the rats were trained in each quadrant once a day. The time for the rats to find and climb up the platform (escape latent period) was recorded on each day and averaged. ${ }^{\#} P<0.01$ compared with sham-operation group; ${ }^{*} P<0.05$ compared with model group.

\section{AS treatment reduces the expression of AB1-42 in the hippocampal region of $A D$ model rats}

To test how AS affects the expression of $\mathrm{A} \beta 1-42$ in hippocampal tissues in rats, immunohistochemistry was used. The data showed that the expression of $A \beta 1-42$ in the hippocampal region of rats in model group was higher than that in sham-operation group (Figure 3). After treatment with AS $(15 \mathrm{mg} / \mathrm{kg}$ and $45 \mathrm{mg} / \mathrm{kg}$ ), the expression of $\mathrm{A} \beta 1-42$ in the hippocampal region was decreased (Figure 3). The results indicate that $\mathrm{AS}$ treatment reduces the expression of $\mathrm{A} \beta 1-42$ in the hippocampal region of $\mathrm{AD}$ model rats.

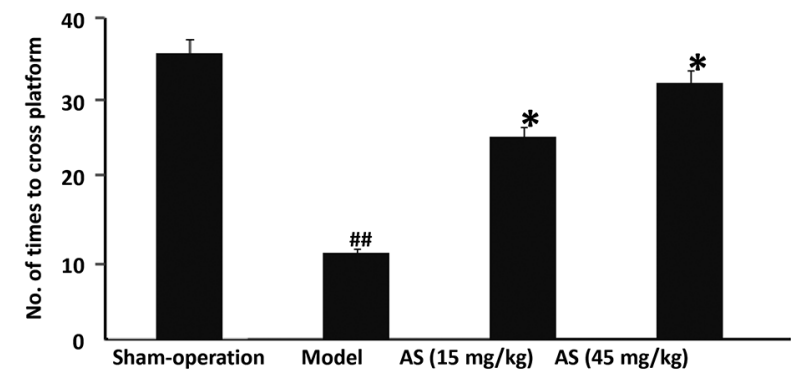

Figure 2. Effect of asiaticoside on the number of times to cross the platform for Alzheimer's disease model rats. Using Morris water maze, spatial search test was performed. On the fifth day, the platform was removed, and the number of times for the rats to cross the original position of the platform within 2 min was recorded. ${ }^{\#} P<0.01$ compared with sham-operation group; ${ }^{*} P<0.05$ compared with model group.
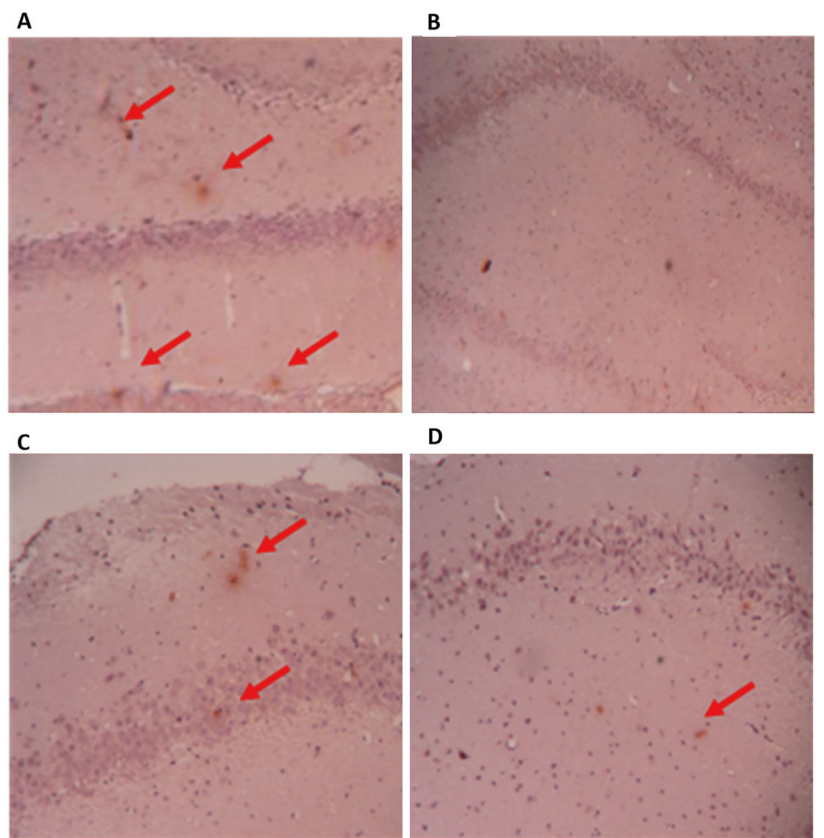

D

Figure 3. Effect of asiaticoside on the expression of AB1-42 in hippocampal tissues from Alzheimer's disease model rats. (A) Model group; (B) sham-operation group; (C) asiaticoside (15 mg/kg) group; (D) asiaticoside (45 $\mathrm{mg} / \mathrm{kg})$ group. Immunohistochemical staining and microscopy were performed to evaluate the levels of AB1-42 in hippocampal tissues (magnification, X200).

\section{Treatment with AS decreases the contents of IL-6 and TNF- $\alpha$ in hippocampal tissues of AD model rats}

To determine the contents of IL- 6 and TNF- $\alpha$ in hippocampal tissues from the rats, ELISA was carried out. The data showed that the contents of IL- 6 and TNF- $\alpha$ in model group were significantly higher than those in sham-operation group $(\mathrm{P}<0.01)$. After treatment with AS $(15 \mathrm{mg} / \mathrm{kg}$ and $45 \mathrm{mg} / \mathrm{kg})$, the contents of IL- 6 and TNF- $\alpha$ were significantly reduced compared with model group $(\mathrm{P}<0.05)$ (Figures $4 \mathrm{~A}$ and $4 \mathrm{~B})$. 
The results suggest that treatment with AS decreases the contents of IL-6 and TNF- $\alpha$ in hippocampal tissues of AD model rats.
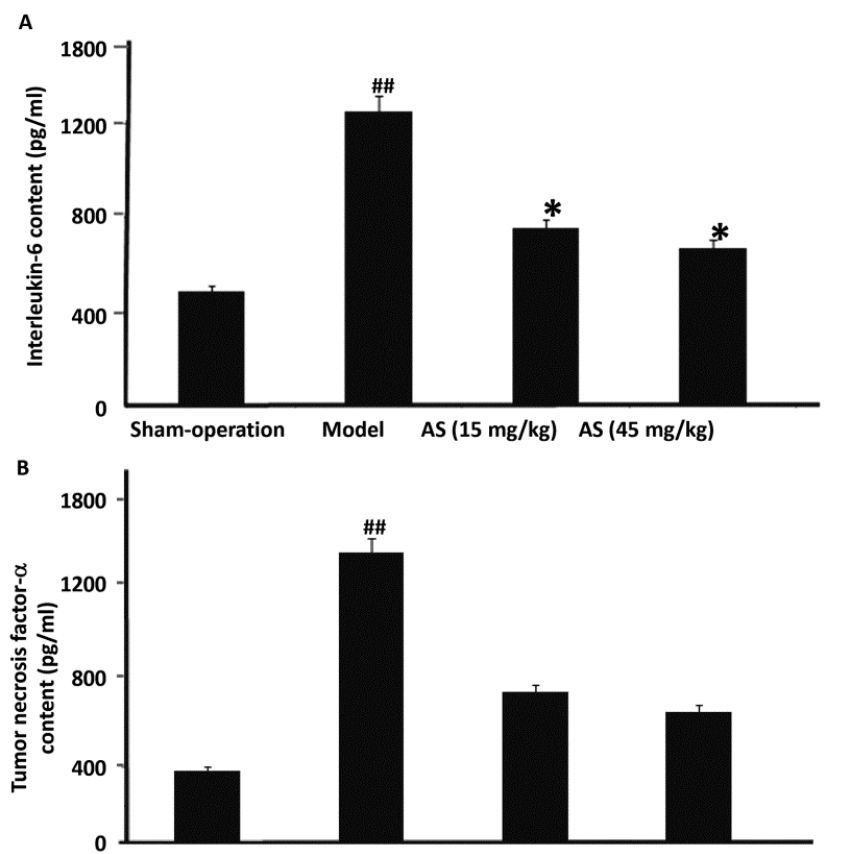

Figure 4. The contents of (A) IL-6 and (B) TNF- $\alpha$ in hippocampal tissues from rats. ELISA was used to determine the contents of IL-6 and $T N F-\alpha .{ }^{\#} P<0.01$ compared with sham-operation group; ${ }^{*} P<0.05$ compared with model group.

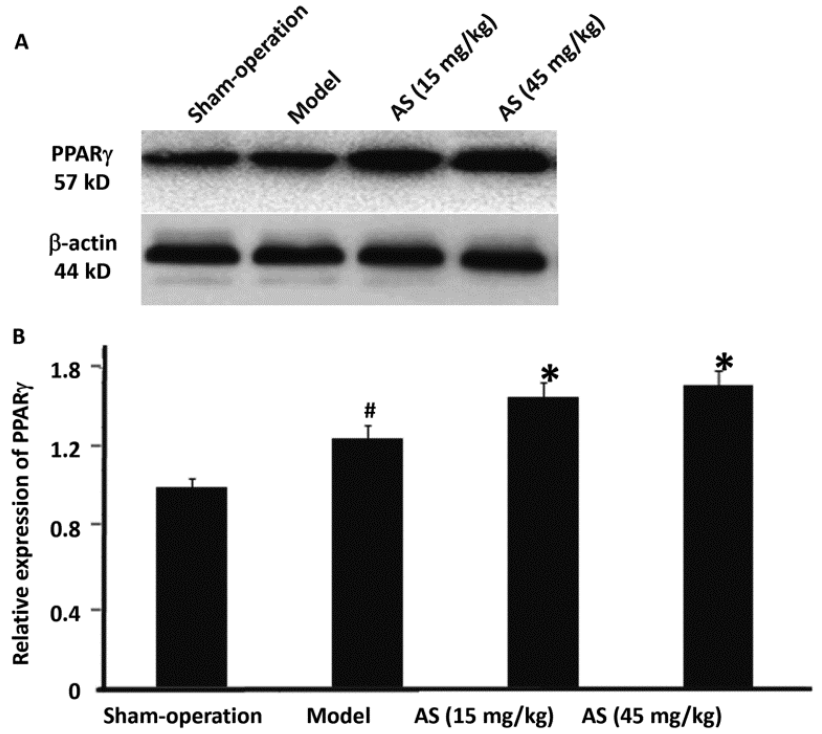

Figure 5. Expression of PPAR protein in hippocampal tissues from rats. Western blotting was employed to measure the levels of PPAR protein. ${ }^{\#} P<0.05$ compared with sham-operation group; ${ }^{*} P<0.05$ compared with model group.

\section{Treatment with AS elevates the protein expression of PPAR $\gamma$ in hippocampal tissues of AD model rats}

To evaluate the expression of PPAR $\gamma$ protein in hippocampal tissues from the rats, Western blotting was employed. The data showed that PPAR $\gamma$ protein expression in model group was significantly higher than that in sham-operation group $(\mathrm{P}<0.05)$. After treatment with AS $(15 \mathrm{mg} / \mathrm{kg}$ and $45 \mathrm{mg} / \mathrm{kg})$, $\operatorname{PPAR} \gamma$ protein expression was significantly increased compared with model group $(\mathrm{P}<0.05)$ (Figures $5 \mathrm{~A}$ and $5 \mathrm{~B})$. The results indicate that treatment with AS further elevates the protein expression of PPAR $\gamma$ in hippocampal tissues of $\mathrm{AD}$ model rats.

\section{Discussion}

It is accepted that inflammatory responses may be one of the factors that lead to the formation and development of brain pathological characteristics in $\mathrm{AD}$ patients. $\mathrm{A} \beta$ peptide is one of the causes of inflammatory responses [7]. Deposition of $A \beta$ may lead to the activation of microglia, start signal reaction pathway, produce inflammatory factors such as IL-1, IL-6 and TNF- $\alpha$, and finally lead to the apoptosis or death of neurons [3]. In the present study, rats are injected with $A \beta 1-40$ in the brain to simulate AD model. The results of the present study demonstrate that AS treatment reduces the latent period and increases the number of times to cross the platform, suggesting that AS improves spatial learning and memory of rats injected with A $\beta 1-42$. Because learning and memory abilities are associated with hippocampal functions, we then detect the deposition of $A \beta 1-42$ in hippocampal tissues. The results show that AS treatment decreases the deposition of $A \beta 1-42$ in hippocampal tissues, suggesting that AS improves the learning and memory abilities of rats by reducing the deposition of A $\beta 1-42$.

Inflammatory responses induced by $\mathrm{A} \beta$ include the release of inflammatory factors TNF- $\alpha$ and IL- 6 , as well as the activation of PPAR $-\gamma$. TNF- $\alpha$ and IL- 6 are mainly produced by central activated microglia and hematogenous macrophages. It is shown that the expression of IL- 6 is elevated in cerebrospinal fluid and cortex of AD patients, and strongly correlated with senile plaque of AD patients, suggesting that IL-6 may participate in the formation of senile plaque [8]. In addition, TNF- $\alpha$ exhibits increased expression in the hippocampus of $\mathrm{AD}$ patients, mediates the intracerebral inflammatory pathological reactions in $\mathrm{AD}$ patients, and promotes the release of other inflammatory factors by microglia [9]. The results of the present study show that the expression of TNF- $\alpha$ and IL-6 is significantly elevated after intracerebral injection of $A \beta 1-40$. In addition, intervention by AS reduces the expression of TNF$\alpha$ and IL-6, suggesting that AS inhibits the production of inflammatory factors.

PPAR $-\gamma$ inhibits the expression of inflammatory factors, and participates in the occurrence and development of AD [10]. After induction by A $\beta$, the levels of PPAR- $\gamma$ in the brain of AD patients are enhanced, suggesting that increase of PPAR- $\gamma$ at early stage of AD inhibits the inflammatory responses caused by $A \beta$. In addition, the level of PPAR- $\gamma$ stops increasing at certain stage, and inflammatory lesions are aggravated [11]. Our results show that PPAR- $\gamma$ protein expression is significantly increased after intracerebral injection of $A \beta 1-42$, but PPAR- $\gamma$ protein expression is not altered in sham-operation 
group, suggesting that PPAR- $\gamma$ expression is stimulated by $\mathrm{A} \beta 1-42$. Of note, intervention by $\mathrm{AS}$ further increases the expression of PPAR- $\gamma$ protein, suggesting that AS may exert its anti-inflammatory effect by inducing the production of PPAR- $\gamma$ protein. In conclusion, AS improves the spatial learning and memory of $\mathrm{AD}$ model rats possibly by inducing antiinflammatory effect and reducing the deposition of $\mathrm{A} \beta$ through the promotion of PPAR $-\gamma$ protein production and inhibition of TNF- $\alpha$ and IL- 6 release. However, inflammation is only one factor that participates in AD. It is worthy of studying whether AS has other mechanisms of action that may induce PPAR- $\gamma$ protein production and anti-inflammation.

\section{Acknowledgements}

This work was supported by Education Office of Sichuan Province (Nos. 13ZB0267 and 14ZA0143) and Technological Office of Sichuan Province (14JC01812014TSX-0065).

\section{Disclosures}

All authors declare no financial competing interests. All authors declare no non-financial competing interests.

\section{References}

1. Cui S, Sun Y, Liu C. Effect of bushenyisui formula on brain tissue apoptosis and Bcl-2 in beta-amyloid protein-induced Alzheimers disease rat models. J Tradit Chin Med 2012; 32: 646-650.

2. Cai Z, Zhao B, Ratka A. Oxidative stress and $\beta$-amyloid protein in Alzheimers disease. Neuromolecular Med 2011; 13: $223-250$.

3. Jiang Q, Heneka M, Landreth GE. The role of peroxisome proliferator-activated receptor-gamma (PPARgamma) in Alzheimers disease: therapeutic implications. CNS Drugs 2008; 22: 1-14.

4. Shie FS, Nivison M, Hsu PC, Montine TJ. Modulation of microglial innate immunity in Alzheimers disease by activation of peroxisome proliferator-activated receptor gamma. Curr Med Chem 2009; 16: 643-651.
5. Liu J, Wang LN, Jia JP. Peroxisome proliferator-activated receptor-gamma agonists for Alzheimers disease and amnestic mild cognitive impairment: a systematic review and meta-analysis. Drugs Aging 2015; 32: 57-65.

6. Hossain S, Hashimoto M, Katakura M, Al Mamun A, Shido O. Medicinal value of asiaticoside for Alzheimers disease as assessed using single-molecule-detection fluorescence correlation spectroscopy, laser-scanning microscopy, transmission electron microscopy, and in silico docking. BMC Complement Altern Med 2015; 15: 118.

7. Cai Z, Hussain MD, Yan LJ. Microglia, neuroinflammation, and beta-amyloid protein in Alzheimers disease. Int $\mathrm{J}$ Neurosci 2014; 124: 307-321.

8. Zhang YY, Fan YC, Wang M, Wang D, Li XH. Atorvastatin attenuates the production of IL-1 $\beta$, IL- 6 , and TNF- $\alpha$ in the hippocampus of an amyloid $\beta 1-42$-induced rat model of Alzheimers disease. Clin Interv Aging 2013; 8: 103-110.

9. Yang YM, Shang DS, Zhao WD, Fang WG, Chen YH. Microglial TNF-alpha-dependent elevation of MHC class I expression on brain endothelium induced by amyloid-beta promotes $\mathrm{T}$ cell transendothelial migration. Neurochem Res 2013; 38: 2295-2304.

10. Mandrekar-Colucci S, Karlo JC, Landreth GE. Mechanisms underlying the rapid peroxisome proliferator-activated receptor-gamma-mediated amyloid clearance and reversal of cognitive deficits in a murine model of Alzheimers disease. J Neurosci 2012; 32: 10117-10128.

11. Landreth GE, Heneka MT. Anti-inflammatory actions of peroxisome proliferator-activated receptor gamma agonists in Alzheimers disease. Neurobiol Aging 2001; 22: 937-944.

\section{*Correspondence to}

Zhuo Zhang

Department of Pharmacology

School of Pharmacy

Southwest Medical University

PR China 